\title{
Hardware/Software Solution Unifying DALI, IBECS, and BACnet Final Report
}

\author{
Ed Koch \\ Akua Controls \\ Francis Rubinstein \\ Sila Kiliccote \\ Lawrence Berkeley National Laboratory
}

December 2004

This work was supported by the Assistant Secretary for Energy Efficiency and Renewable Energy, Building Technologies Program, of the U.S. Department of Energy under Contract No. DE-AC03-76SF00098. 


\section{Table of Contents}

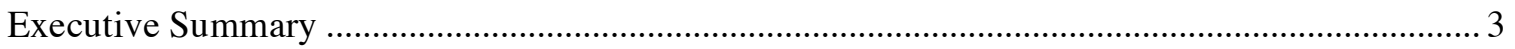

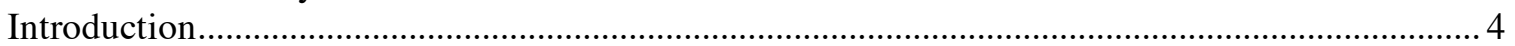

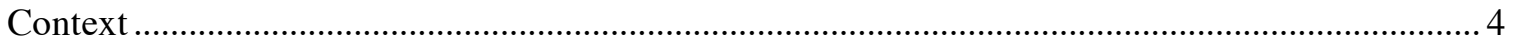

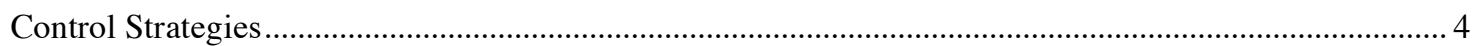

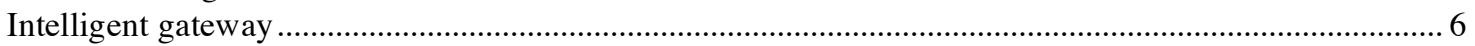

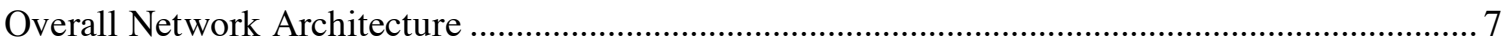

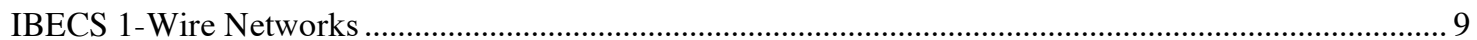

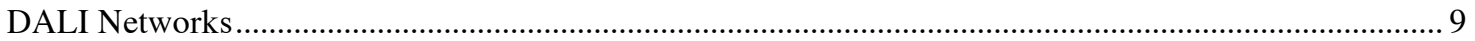

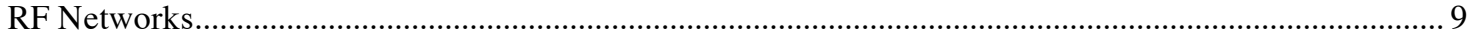

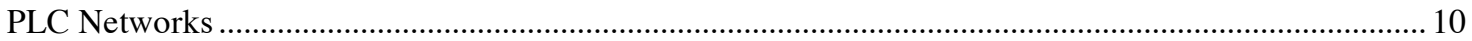

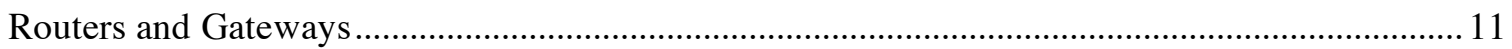

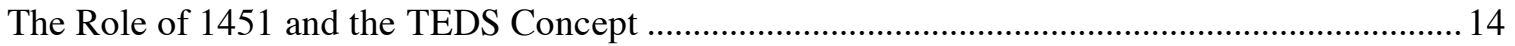

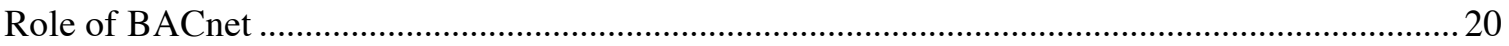

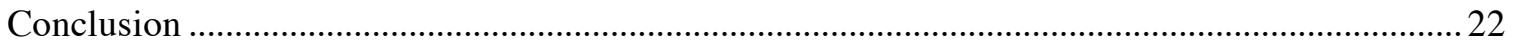

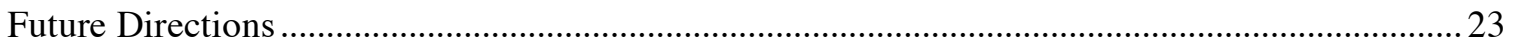

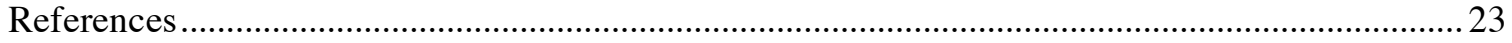




\section{List of Figures}

Figure 1. Generalized system diagram showing relationship between the WAN, LAN and multiple DANs operating different subnets throughout a facility.................... 8

Figure 2. System diagram showing how encapsulated DAN packets are transported on the Ethernet LAN and routed to different DAN subnets that run the same communication protocol.

Figure 3. System diagram showing how encapsulated DAN packets are transported on the Ethernet LAN by gateways able to perform both protocol and data model translations.

Figure 4. Three different subnets, 1 -wire, PLC, and RF, that communicate via their respective protocols to network-specific controllers that embed a TEDS.

Figure 5. A simple DALI gateway, with virtual TEDS and STIMs residing in the gateway rather than in the DALI device itself.

Figure 6. Generalized system diagram showing the NCAP (Network Capable Applications Processor) on the IBECS 1451 gateway and how communications between the NCAP and the different network blocks is mediated through a standardized 1451 interface.

Figure 7. Generalized system diagram showing how a BACnet aware application can control both BACnet IP capable devices directly on the data LAN as well as networked devices on DALI, IBECS, PLC, or RF networks via the IBECS BACnet gateway and the 1451 standardized interface.

Figure 8. Generalized system diagram showing how BACnet and 1451-aware application can control both BACnet IP and 1451.1 devices directly on the data LAN as well as networked devices on DALI, IBECS, PLC, or RF networks via the IBECS BACnet gateway and the 1451 standardized interface. 


\section{Executive Summary}

Many lighting control companies have robust lighting control systems with functional strategies for the office level controls. What the industry lacks is the efficient integration of local controls with building controls and automation systems in order to utilize sensory data. LBNL proposes a framework for a gateway with a level of embedded intelligence, linking various device area networks (DANs) to building control systems.

The goal of a lighting control system is to provide additional control capabilities to the occupants, and to implement localized control strategies such as daylighting or occupancy sensing for increasing energy efficiency. These strategies work locally, that is, all the "inputs" required for successful operation of the lighting system are obtained locally, at the point of use. "Global" sensor data acquired at the building level is not required or necessary.

Many control opportunities open up when one considers integrating local control with building-wide control and automation, which is the purpose of this report.

The successful integration of office and building level control systems can:

1) improve the demand responsiveness of a facility

2) improve lighting and building control operational efficiency by implementing daylighting and other sensor-based control strategies

3) eventually allowing lighting sensor data to help inform "intelligent agents" that monitor a building's sensor information for life safety applications.

To provide these additional capabilities, the report proposes an intelligent gateway that would intermediate between local office lighting controls and building-level control and automation systems. The intelligent gateway uses IEEE 1451 Standard on Sensor and Actuators as the basis for standardizing communications between the network and building equipment and sensors. IEEE 1451 develops the concept of the TEDS table (Transducer Electronic Data Sheet) which provides a standardized way to electronically document the capabilities of any piece of networked building equipment or sensor. This electronic document can be read by application software to identify the capabilities of any connected device and thus control it.

The intelligent gateway concept proposed in this report allows the above benefits to be realized cost-effectively by building on existing communications protocols that are already being used for building automation today. The proposed system is networkneutral. That is, it will function regardless of which networking protocol is used. It is also manufacturer neutral in that the TEDS concept provides a standardized manufacturerneutral format for sensors and devices which allows devices from different manufacturers to cooperate with one another to accomplish useful things in buildings.

The proposed gateway acts as a translator for DANs enabling them to talk to each other and with a building control system. Just like a PC recognizing a mouse as soon as it is plugged in, the gateway will recognize devices with embedded or virtual TEDS. This presents a truly "plug and play" capability for the building control systems. As a result, 
sensory data can be automatically calibrated, collected and utilized with minimal labor for effective and efficient building controls.

\section{Introduction}

The goal of this project was to investigate broader building-level systems/strategies that enable further energy savings and control. This project investigated the potential savings offered by broader centralized control features and the potential advantages they may add to this system through such features as addressability and load shedding. This report documents the results of LBNL's work in this area.

This report focuses on building-level systems and strategies and a multi-protocol gateway solution that is indifferent to the specific choice of lighting control/communications technique used to control the office lighting. The elegance of the IEEE 1451 intelligent gateway proposed in this report is that the overall building communications system should work regardless of whether the office lighting is controlled by DALI, UPB, IBECS, ZigBee or any other accepted communications protocol.

\section{Context}

In this report, we make the case for integrating office lighting control with building-wide environmental controls and show the application benefits of such an integration.

\section{Control Strategies}

At the office level, the goal of the lighting control system is to provide additional control capabilities to the occupants, and to implement localized control strategies such as daylighting or occupancy sensing for increasing energy efficiency. These strategies work locally, that is, all the "inputs" required for successful operation of the lighting system are obtained locally, at the point of use. "Global" sensor data acquired at the building level is not required or necessary.

More control opportunities open up when one considers integrating local control with building-wide control and automation, which is the purpose of this report.

The successful integration of office and building level control systems can:

4) improve the demand responsiveness of a facility

5) improve lighting and building control operational efficiency by implementing daylighting and other sensor-based control strategies

6) eventually allowing lighting sensor data to help inform "intelligent agents" that monitor a building's sensor information for life safety applications.

Data can be collected from global sensors and from utility pricing signals to help make lighting, envelope and HVAC control systems more effective at exploiting available daylight or making building control systems responsive to power demand requirements. Integrating local lighting systems with building-level controls can provide occupancy 
status throughout a facility for life safety applications. In this advanced concept, an intelligent agent running on a network-connected server would analyze the occupancy status from multiple distributed occupant sensors to immediately inform an evacuation plan in the event of a building emergency (fire, physical attack, etc).

For example, to most efficiently implement demand responsive lighting requires knowledge of the real price of electricity going to the facility. This global input is not ordinarily available to a local control loop. Rather it must be supplied globally by the building automation or automated metering system that operates at the whole building level. In this load shedding scenario, the lighting control system would operate based on local sensors most of the time, but during power emergencies, local lighting loads would be shed (reduced in intensity either by switching or dimming) when commanded to upon receiving a signal based on the globally available cost of electricity.

Advanced control strategies such as daylighting generally use local lighting sensors to control overhead lighting in response to available daylight. But daylighting control system often can operate more reliably when local sensor information is supplemented by global sensor data, such as the external horizontal irradiance or illuminance.

According to the California Energy Commission's Demand Analysis Office findings, commercial lighting is $11-20 \%$ of peak electrical load in a typical commercial building. Considering that in the summer each $3 \mathrm{~kW}$ of lighting adds about $1 \mathrm{~kW}$ of additional burden to the air conditioning system, lighting loads contributes about $4 \%$ indirectly. In other situations, non-lighting systems (such as HVAC) may be able to operate more efficiently if they can acquire occupancy data from occupant sensors that normally control local lighting. In this case, a large HVAC zone would optimize operation based on an analysis of the actual real time occupancy as detected by occupancy detectors. For example, in addition to switching lights off where people are not present, occupancy patterns can be directly fed back to the HVAC use an economizer to modulate the fresh air intake rate in areas with low density or areas no longer occupied. The shades in unoccupied areas can be adjusted to cut off the direct sunlight, lowering solar heat gain. Of course, for this to work, the data from the occupant sensors must be available not only to the local light switch but also to a building-wide communications network that can in turn communicate with other building control systems.

Integration of occupancy, illuminance sensors and imaging sensors with building control systems can yield tremendous non-energy benefits. Sensors that are useful for lighting control are also potentially very useful for life safety applications. If each office is equipped with an occupancy sensor and this data can be gathered, analyzed and acted upon by an automation system, building life safety can be significantly improved. The uniting theme here is that if sensor information can be made globally available to any system needing that information, then building operation, efficiency and life safety can be significantly enhanced at lower added cost since the basic sensors are already necessary for energy efficiency. Getting double and triple duty from local sensors greatly improves the value proposition for advanced controls and goes a long way to justifying their added cost. 


\section{Intelligent Gateway}

The goal of the building level control system is to deliver amenities to each occupant without compromising energy efficiency and system integrity. Building level control system design, installation and implementation is an extremely challenging problem. Various trades are involved. A lack of knowledge in one area delays the completion of the project and has adverse effects on the performance of the entire system. The "intelligence" of the building control systems does not only come from clever use of sensory data for the development of control strategies, but also the intelligent decisions made during the design, integration, installation, configuration and commissioning of each system. The cost of the systems can be greatly minimized if some of the initial labor-intensive tasks, such as the initial configuration and commissioning of the system are automated.

To integrate local lighting with building control systems requires adding networkable intelligent gateways to building automation systems. An intelligent gateway is a networking device much like WiFi routers that are proving so useful adding wireless connectivity to residential and commercial computer networks (LANs). Gateways are usually connected to the Ethernet on one side and to the local building LAN on the other. (For WiFi routers, Ethernet is usually provided on the gateway input and the output is the wireless LAN that interconnects all local computers and allows them to pass information to the Internet through the router.). In building controls applications, the Ethernet is also present at one end but the output will be a wired building control network (such as Modbus or DALI) or a wireless network (such as the ZigBee protocol).

The gateway is a necessary and critical component of a successfully integrated control system since it connects the Internet on one side to a building specific network on the other. Gateways are analogous to freeway interchanges. They allow cars (data packets in our case) to transfer from local roads (building-specific networks) to the freeway (the Internet) and back again. The difference is that interchanges merely transfer cars from one road to another - an intelligent gateway actually translates data packets from one protocol to another (for example from the TCP/IP protocol used for Ethernet to a building-specific network protocol).

In this report, a gateway that translates signals between device area networks (DAN) and building control systems is proposed. A level of intelligence is embedded so that the gateway framework can undertake configuration and commissioning tasks by recognizing the devices, their physical limitations and their tasks. It is proposed that the gateway utilizes Transducer Electronic Data Sheets (TEDS) to enable the building control system to detect and automatically configure sensors. This technology stores data sheets electronically. Thus, it eliminates manual data entry, improves accuracy with detailed calibration information, and reduces configuration time.

This framework relies on the adoption of IEEE 1451.2,3, and 4 standards. IEEE 1451 is a family of standards for connecting smart transducers to networks, which introduces the 
concept of TEDS. The application of this concept has not been expanded to the lighting sector yet and has been limited to industrial and environmental monitoring applications. The following protocols and standards will be considered in this report:

Table 1. Standards and Protocols for Building Controls

IBECS 1-Wire LBNL's application of an enhanced 1-wire network to lighting controls and building automation.

RF New and emerging wireless networks that use radio frequency PLC Power Line Carrier (and other PL communications methods)

IEEE 1451 Standard for Sensors and Actuators

BACnet Building Automation Controls

\section{Overall Network Architecture}

Figure 1 below shows an example of a hierarchical network framework that can be used for lighting control applications.

For the purposes of this analysis the network is decomposed into three main segments titled:

- WAN - Wide Area Network, IP data network whose scope spans large geographic areas.

- LAN - Local Area Network, IP or data communications network whose scope is within the facility where the control system exists.

- DAN - Device Area Network, refers to the control network in which telemetry and control devices are installed. 


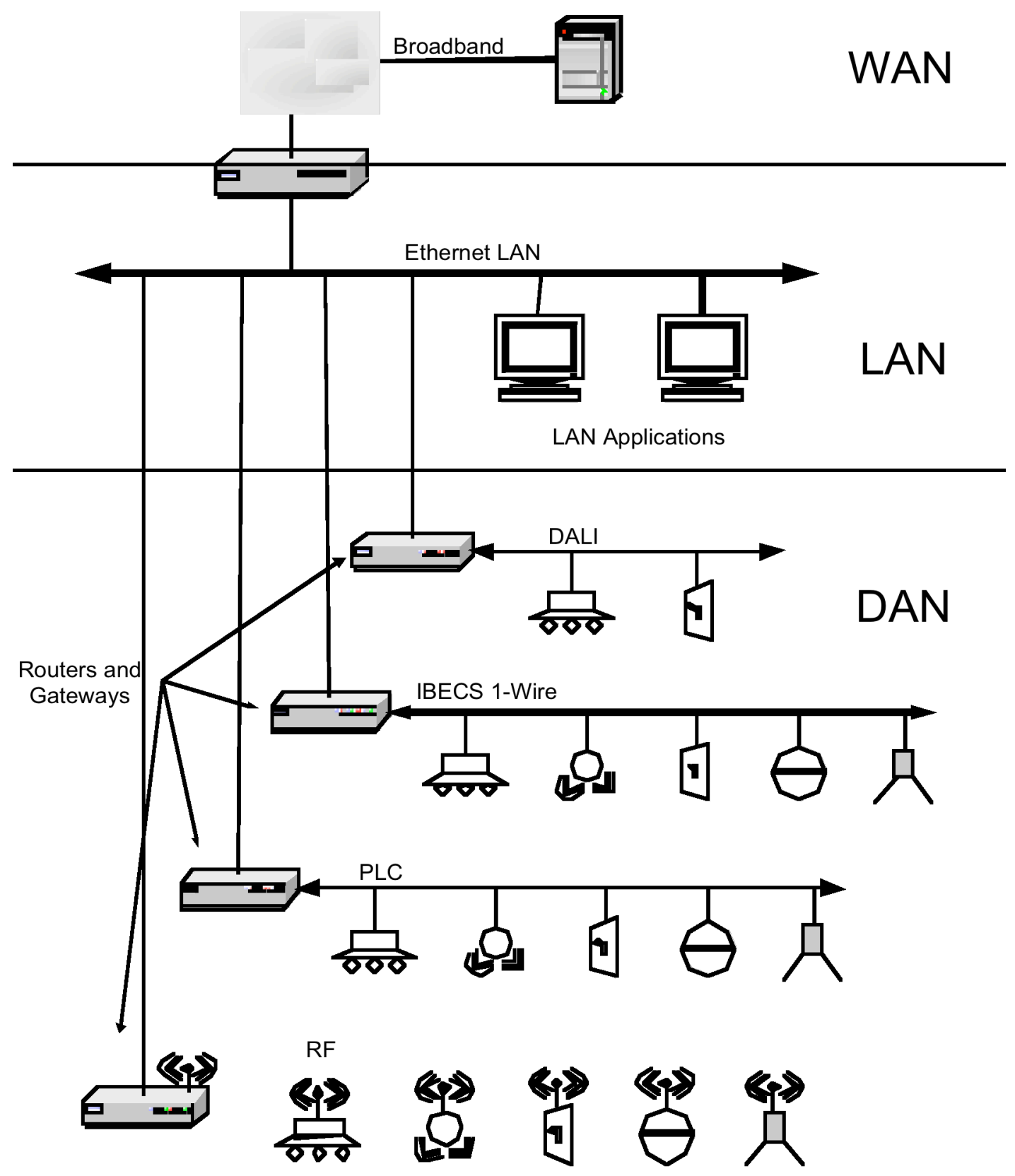

Figure 1. Generalized system diagram showing relationship between the WAN, LAN and multiple DANs operating different subnets throughout a facility.

For the purposes of this report, the WAN segment of this hierarchy is not analyzed beyond the fact that it includes the Internet and obviously plays an important role in any communications network.

The LAN segment of the network is typically used for data communications and is the main communications infrastructure used by the system-wide building control applications. As such, these applications must communicate in some way with the building control actuators and sensors. As shown in the diagram, this is done via 
gateways or routers that link the DAN networks with the LAN networks. The integration of the communications technologies between the LAN and the DAN is only part of what is required. In addition there must be some sort of semantic integration between the applications on the LAN and the devices on the DAN. This typically means that the data models used by the LAN applications must be consistent with the data models used by the DAN devices. Sometimes this can be accomplished directly between a LAN application and a DAN device by doing protocol translation, but often it is necessary to insert an intermediary agent such as a gateway that can perform some sort of data translation and mapping between the LAN application and the DAN device's interface. BACnet is an emerging standard in the building automation industry for allowing control applications on the LAN to communicate and use devices on the DAN. It includes both semantic and protocol level support. Of particular note in the context of IBECS is the BACnet IP standard, which allows BACnet to operate over IP networks. BACnet IP allows BACnet applications running on the LAN to more easily integrate with the devices on the DAN via gateways and routers as shown in Figure 1.

The DAN segment of Figure 1 shows examples of some of the most important types of network technologies for lighting control. These are discussed in more detail in the sections below.

\section{IBECS 1-Wire Networks}

IBECS 1-Wire Networks are an adaptation of Dallas Semiconductors 1-Wire technology. Its main benefit is its low cost, and readily available and simple components. LBNL has done some useful research into extending its range and making it more robust. Before it can be commercially deployed, there still needs to be some additional research and field tests to determine its operating constraints and to develop best practices to reduce installation problems and costs. IBECS 1-Wire requires wiring and is probably best suited for new construction or major renovation projects.

\section{DALI Networks}

DALI was developed specifically for lighting control and has begun to gain acceptance in the lighting industry in recent years. Over the last year, the National Electrical Manufacturers Association (NEMA) Joint Sections Committee on DALI in collaboration with the PIER LRP under Project 5.4 has been developing a controls "overlay" to the original DALI protocol that will allow control devices to peacefully co-exist on a DALI ballast network. DALI's main benefit is simplicity of design and industry acceptance by US ballast manufacturers. DALI requires wiring and is probably best suited for new construction or major renovation projects.

\section{RF Networks}

Radio frequency communication has evolved rapidly over the last five years and costs are poised to drop dramatically. The most common application for RF wireless today is networking for computing applications. As wireless extends its reach from computer 
networking to appliances and building equipment, two strategies have emerged: IEEE 802.11b (aka WiFi), and Bluetooth. ZigBee is a third method that has found support from electronics giants, such as Philips and Honeywell that are entering the wireless building controls market. The following table (Table 1) summarizes the key technical characteristics of the systems.

Table 2. Characteristics of three wireless communication standards

\begin{tabular}{|c|c|c|c|}
\hline & "WiFi" & "Bluetooth" & "Zigbee" \\
\hline Effective & $500 \mathrm{~m}$ & $10 \mathrm{~m}$ & $30-75 \mathrm{~m}$ \\
\hline $\begin{array}{l}\text { Distance } \\
\text { Frequency }\end{array}$ & $2.4 \mathrm{GHz}$ & $2.4 \mathrm{GHz}$ or & 2.4 GHz or \\
\hline Applicable & IEEE $802.11 \mathrm{~b}$ & IEEE 802.15.1 & $\begin{array}{l}\text { IEEE } \\
802.15 .4\end{array}$ \\
\hline $\begin{array}{l}\text { Spread } \\
\text { Spectrum }\end{array}$ & $\begin{array}{l}\text { Direct sequence } \\
\text { (DHSS) }\end{array}$ & $\begin{array}{l}\text { Frequency } \\
\text { hopping } \\
\text { (FHSS) }\end{array}$ & $\begin{array}{l}\text { Direct } \\
\text { sequence } \\
\text { (DHSS) }\end{array}$ \\
\hline Data Rate & $11 \mathrm{Mbps}$ & $721 \mathrm{kbps}$ & $\begin{array}{l}20 \mathrm{kbps}- \\
250 \mathrm{kbps}\end{array}$ \\
\hline
\end{tabular}

\section{PLC Networks}

All existing powerline carrier systems (PLC) use electronic wiring devices to send information over $120 \mathrm{~V}$ and $277 \mathrm{v}$ electrical power distribution systems of a building by superimposing a high frequency carrier signal on top of the fundamental power frequency. In a PLC system, a generator is used to impose a 1 to $4 \mathrm{~V}$ high frequency signal on top of the existing $60 \mathrm{~Hz}$ voltage sine wave. The superimposed signal is generally in the $80-200 \mathrm{kHz}$ range with some older systems operating at 19,500 Hz or higher. With PLC, control signals are transmitted from controller to actuators over the inplace electrical power wiring. The problem with PLC is that some building equipment can "absorb" the injected PLC signal preventing proper operation. For example, electronic ballasts that are capacitive in nature (which describes most commerciallyavailable electronic ballasts) can absorb the signal from a PLC transmitter. As a result, the signal becomes too weak to be "heard" by the receiver (like a timeclock) connected to the powerline.

Several technologies and products exist to provide high bandwidth, Internet speed, communication via hard-wired, powerline, and wireless approaches, but these are far too expensive to be used for energy efficiency or conservation applications. A number of companies (X10, CEBUS, LonWorks, Intellon, Itran and Inari) have developed less expensive, low bandwidth PLC based communication technologies, but these are barely reliable enough to be deployed within the electrically simplistic environment of residential structures and far too unreliable to be deployed in the commercial three-phase environment. 
PLC holds the promise of using existing power lines for communication and may be appropriate for both new building and retrofit applications. There are a number of companies that offer PLC technology for control applications including Echelon, ITRAN, Domosys, and PCS Lighting just to name a few. In addition LBNL has done some useful research into using PLC for lighting control [2]. Issues with current PLC offerings include a distinct lack of standards and the relatively high cost of the technology, which makes it difficult to justify for lighting controls. In addition, PLC tends to be unreliable and more difficult to use in commercial applications. The new PLC technology being developed by PCS Lighting holds some promise as being a low cost option for PLC communications and merits further investigation.

\section{Routers and Gateways}

As shown in Figure 1, each of the devices in the DAN typically interfaces to the LAN using some sort of gateway or router. Routers typically move native DAN packets over LANs without doing any significant protocol translation or data mapping. An example of this type of routing can be found in EIA-852 that is a standard that allows the tunneling of DAN type packets over IP networks. BACnet has a similar standard that allows the tunneling of BACnet packets over IP networks. It should be noted that this is different than BACnet IP, which uses IP as the BACnet transport protocol. Tunneling routers are depicted in Figure 2.

Figure 2 shows two Control Subnets in the DAN segment. Each of the Control Subnets $\mathrm{A}$ and $\mathrm{B}$ are composed using the same communications protocol. The role of the Tunneling Routers are to take DAN packets from a Control Subnet (i.e. Subnet A), encapsulate them into an IP packet and forward them on to other Tunneling Routers on the IP LAN where they are subsequently extracted from the IP packet and transmitted onto the other DAN Subnet (i.e. Subnet B). The IP protocol used to encapsulate the DAN packets is known as the "tunneling protocol". EIA-852 is an example of a tunneling protocol for DAN type packets. Depending upon the requirements of the DAN protocol, the tunneling protocol may be very simple or it can be quite complex. Complexities usually arise when there are timing constraints of the DAN protocol that are difficult to maintain in IP networks. 


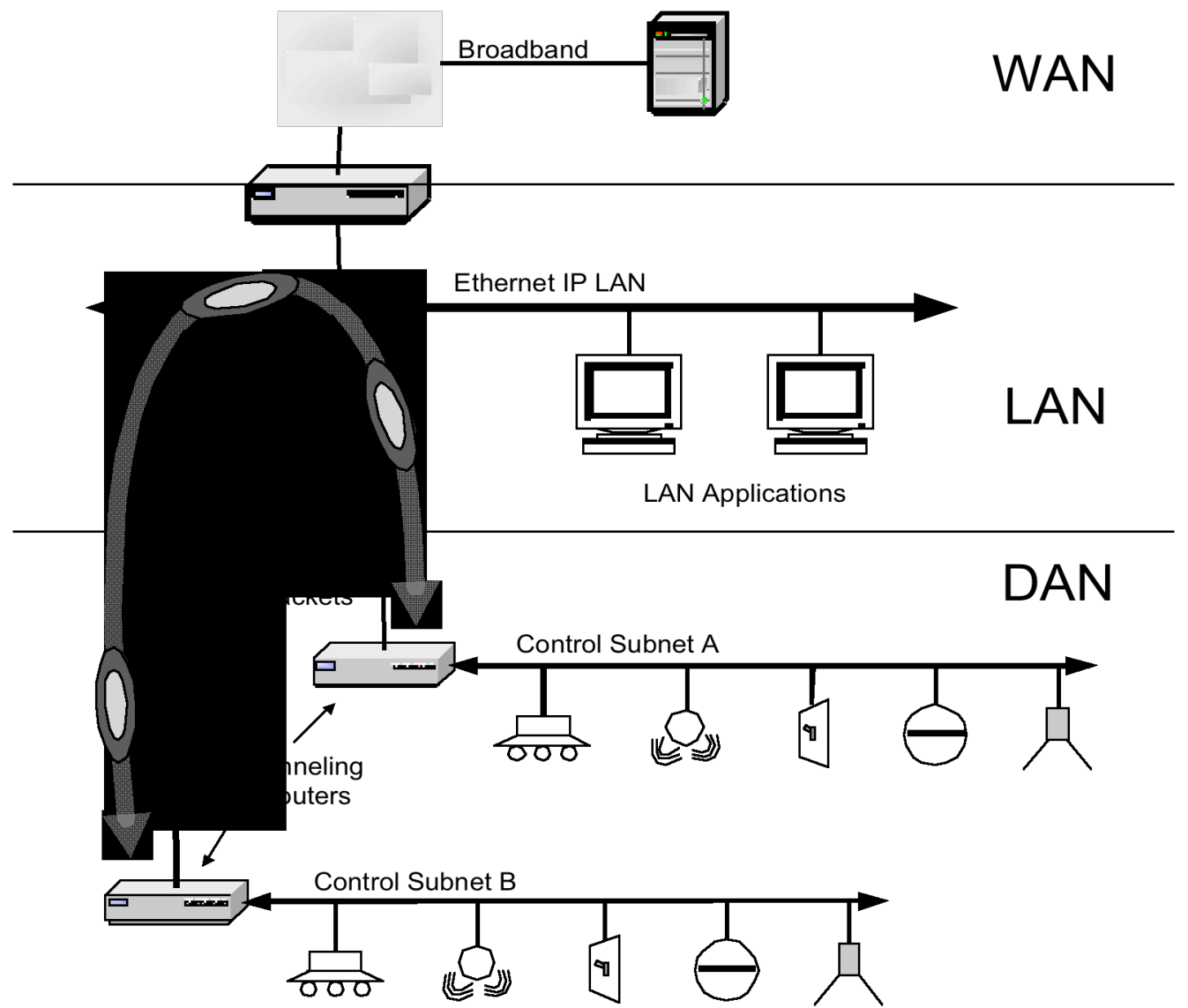

Figure 2. System diagram showing how encapsulated DAN packets are transported on the Ethernet LAN and routed to different DAN subnets that run the same communication protocol.

The scenario discussed above assumes that the Control Subnets A and B use the same Dan protocol, but it is possible to transmit DAN packets between two Subnets that do not use the same DAN protocol. In this case, the routers involved perform both protocol translation and packet tunneling between the DAN Subnets. If the differences between the DAN protocols are significant, it may not be possible to perform a simple protocol translation.

Tunneling DAN packets in this fashion is an effective way to utilize the LAN as a backbone or medium for expanding the DAN communications, but it can also be used to communicate with LAN applications. For this to be possible, the LAN applications must speak both the tunneling protocol and the specific DAN protocol to communicate with each Subnet. The need for the LAN application to speak so many different DAN protocols will make it more difficult to interface with a variety of DAN Subnets. In addition, it must also adopt the data models and interfaces of each device on each DAN segment.

An alternative to using tunneling routers is to use a gateway that communicates with the LAN applications using a single standardized protocol and data model that translates the 
various DAN protocols and data models to be used by the LAN applications. This is depicted in Figure 3 below.

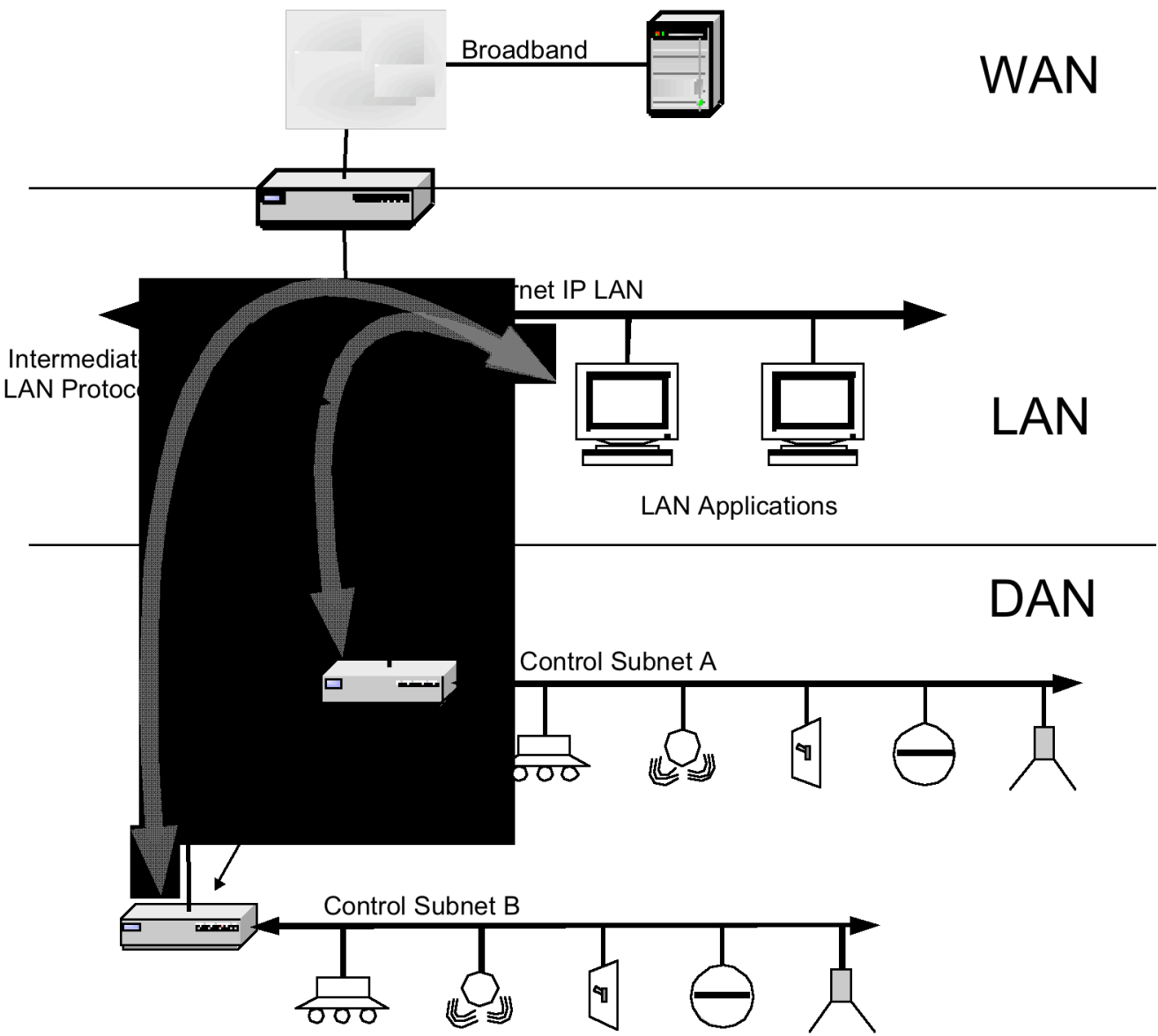

Figure 3. System diagram showing how encapsulated DAN packets are transported on the Ethernet LAN by gateways able to perform both protocol and data model translations.

The gateway approach has the advantages of defining a single protocol and data model for LAN applications thus reducing the number of standards and protocols that LAN applications must adopt. If a suitable LAN protocol and data model can be chosen, there is a much better chance for industry adoption and interoperability between a variety of applications. This is where 1451 and BACnet can play a role as will be discussed in subsequent sections.

The gateway depicted in figure 3 is responsible for facilitating communications between LAN applications and DAN Subnets. It does this by performing both protocol and data model translations. In addition to facilitating communications, the gateway can also perform local logic and functions of its own. If so enabled, it can be programmed to interface with the devices on the DAN to read values and issue commands. Thus, the system wide logic that may typically be executed at centrally located LAN applications can be distributed to a multitude of gateways. This may be done for a variety of reasons including: 
- Reduce network traffic on the LAN.

- Reduce latencies in control loops that utilize the devices on the DAN.

- Eliminate single point of failures in the LAN applications.

- Provide for redundancy in the control algorithms.

Typically, there are three levels of programmability that may be utilized to provide control functions in a gateway.

1. The first level is a fixed set of functionality built into the gateway that has been parameterized such that the programmability of the gateway is accomplished simply by using an API to set the parameters from the LAN application. This is the simplest method if the applications and functionality can be defined in advance. It suffers from being somewhat inflexible.

2. The second level is to design the gateway with the ability to run interpreted programs that can be loaded into it from the LAN applications. This provides for more flexibility, but can be more difficult to use. The programming language can be a simple scripting language such as Tcl or something as complete as Java.

3. The final level is to allow for native code to be developed and loaded into the gateway. This has the maximum flexibility, but is by far the most difficult to execute and the most prone to introducing bugs that require the gateway to be replaced. This level of programming is rarely made available to LAN applications.

\section{The Role of 1451 and the TEDS Concept}

IEEE 1451 is a suite of standards, each aimed at standardizing communications for smart transducers. The diagrams in this section show a more detailed breakdown of the various roles of 1451 in a unified IBECS framework.

The Transducer Electronic Data Sheet (TEDS) provides a standardized way to electronically document the capabilities of a device. This electronic document can be read by applications that wish to use the device and thus the capabilities of the device can be discovered at run time. In other words, the application can determine the capabilities of a new device even if it has never seen the type of device before. The TEDS concept, which is developed and refined in the 1451.0, 1451.2 and 1451.4 standards, provides a robust mechanism to identify and exploit the capabilities of all the control devices on the network. The fully developed TEDS consists of three components: the Basic TEDS, the Standard and Extended TEDS, and the User Area. As the names imply, the basic TEDS would be common to all devices of a particular class (say, occupancy sensors). The Standard and Extended TEDS would be more manufacturer specific and provide manufacturers with product differentiation. The User Area would store information local to the installation, such as the GPS coordinates of the switch, for example.

The tables below show examples of TEDS for a simple dimmable ballast, photosensor and an occupancy sensor. These examples are purely illustrative. Eventually, 
manufacturers will craft the TEDS format to accommodate their different devices in a mutually agreed-upon format.

Table 3. Example TEDS for a Dimmable Ballast

\begin{tabular}{|c|c|c|}
\hline \multirow{4}{*}{ Basic TEDS } & Manufacturer ID & 33 \\
\hline & Model Number & 7171 \\
\hline & Version Letter & $\mathrm{C}$ \\
\hline & Serial Number & XYZ1234 \\
\hline \multirow{6}{*}{$\begin{array}{l}\text { Standard and Extended } \\
\text { TEDS }\end{array}$} & Calibration Date & Jan 15,2000 \\
\hline & Number of light levels & 255 \\
\hline & $\begin{array}{l}100,90,80,75,25,15, \\
10\end{array}$ & $\begin{array}{l}254,230,220,150,45,21, \\
21\end{array}$ \\
\hline & Factory Min & 75 \\
\hline & Default Fade Up & Fast \\
\hline & Default Fade Down & Slow \\
\hline \multirow{8}{*}{ User Area } & Switch Location (True) & GPS coordinates \\
\hline & Switch Location (Local) & Building 90 Room 304 \\
\hline & Max & 250 \\
\hline & Min & 45 \\
\hline & Group Membership & 1, 3, "room 3","emergency" \\
\hline & Fade Up & $\overline{\text { Med }}$ \\
\hline & Fade Down & Default \\
\hline & Service date & Jan 15,2004 \\
\hline
\end{tabular}

Table 4. Example TEDS for an Occupancy Sensor

\begin{tabular}{|lll|}
\hline \multirow{4}{*}{ Basic TEDS } & $\begin{array}{l}\text { Manufacturer } \\
\text { ID }\end{array}$ \\
\cline { 2 - 3 } & Model Number & 7173 \\
\cline { 2 - 3 } & Version Letter & B \\
\cline { 2 - 3 } & Type & Ultrasonic \\
\cline { 2 - 3 } & Serial Number & XYZ6789 \\
\hline $\begin{array}{l}\text { Standard and Extended } \\
\text { TEDS }\end{array}$ & Calibration Date & Jan 15, 2000 \\
\cline { 2 - 3 } & Number of states & 2 \\
\cline { 2 - 3 } & Sensor Range & $50 \mathrm{~m}$ \\
\cline { 2 - 3 } & $\begin{array}{l}\text { Default } \\
\text { Sensitivity }\end{array}$ & High \\
\hline
\end{tabular}




\begin{tabular}{|lll|}
\hline & Default Delay & 10 \\
\hline \multirow{4}{*}{ User Area } & Switch Location & Conference Room \\
& & 304 \\
\cline { 2 - 3 } & Sensitivity & Low \\
\cline { 2 - 3 } & Delay & 5 \\
\cline { 2 - 3 } & Service date & Jan 15, 2004 \\
\hline
\end{tabular}

Table 5. Example TEDS for a Control Photosensor

\begin{tabular}{|c|c|c|}
\hline \multirow{5}{*}{ Basic TEDS } & Manufacturer ID & 33 \\
\hline & Model Number & 7171 \\
\hline & Version Letter & $\mathrm{C}$ \\
\hline & Type & Silicon phodiode \\
\hline & Serial Number & XYZ1234 \\
\hline \multirow{10}{*}{$\begin{array}{l}\text { Standard and Extended } \\
\text { TEDS }\end{array}$} & Calibration Date & Jan 15,2000 \\
\hline & $\begin{array}{l}\text { Color Correction } \\
\text { (Fl1/D65) }\end{array}$ & $15 \%$ \\
\hline & $\begin{array}{l}\text { Color Correction } \\
\text { (Fl1/D55) }\end{array}$ & $10 \%$ \\
\hline & Max Output (5V) & $1250 \operatorname{lux}$ \\
\hline & Range1 Max & 100 \\
\hline & Range2 Max & 500 \\
\hline & Range3 Max & 1250 \\
\hline & Symmetry Type & II \\
\hline & FOV Parallel & 15 \\
\hline & FOV Perpendicular & 30 \\
\hline \multirow{3}{*}{ User Area } & Switch Location & $\begin{array}{l}\text { Conference Room } \\
304\end{array}$ \\
\hline & Range & 2 \\
\hline & Service date & Jan 15, 2004 \\
\hline
\end{tabular}

Ideally, the TEDS is embedded within the devices on the network. This is illustrated in Figure 4 with devices on an IBECS 1-Wire, PLC, and RF network. The requirements for embedding a TEDS in a transducer are quite minimal and it is expected that the devices that reside on these networks will be capable of doing this. It should be noted in this scenario that although these networks are currently not compatible with the electrical interface that was specified in the original 1451.2, there are efforts underway in 1451.0, 1451.2, 1451.4, and 1451.5 to support general serial and RF interfaces like those shown in figure 3. The concept of a TEDS and its functionality is applicable regardless of the physical interface on the IBECS network. 
IBECS 1-Wire Network

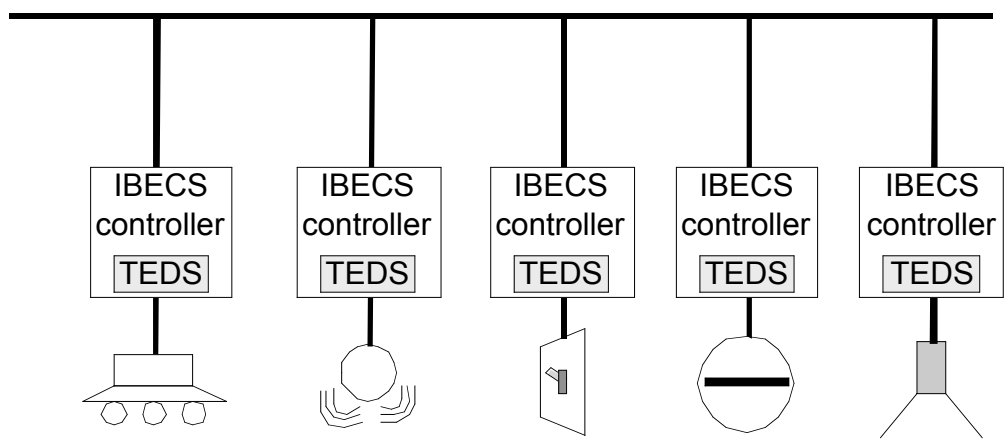

PLC Network

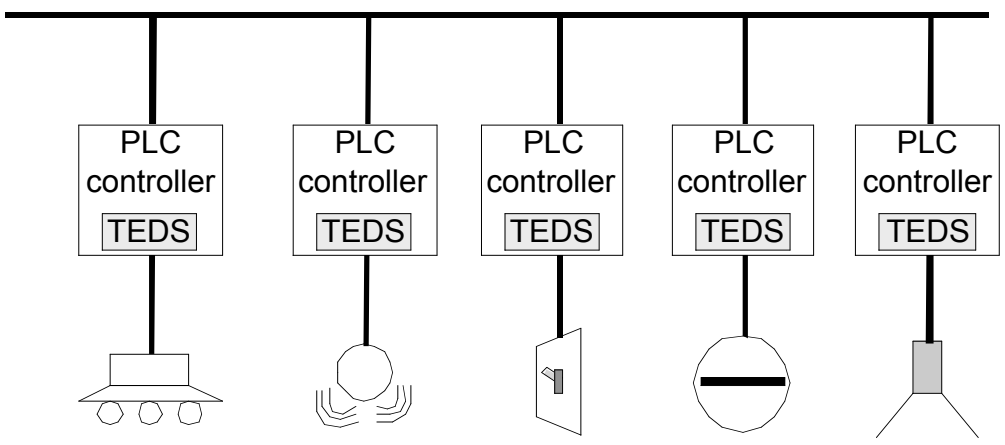

RF Network
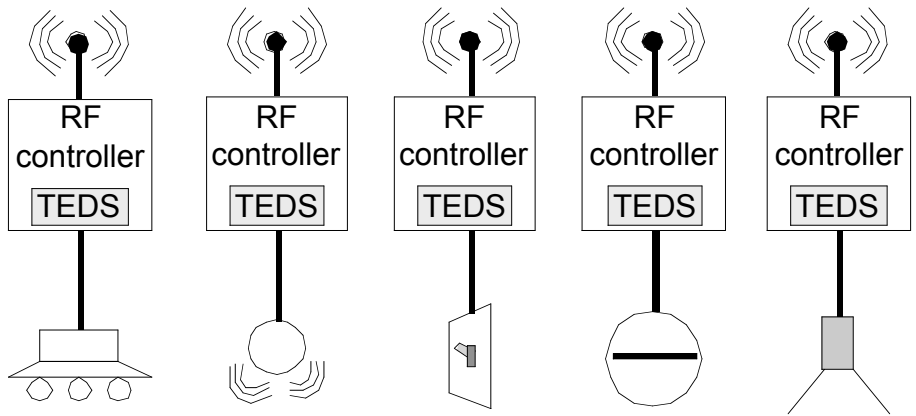

Figure 4. Three different subnets, 1-wire, PLC, and RF, that communicate via their respective protocols to network-specific controllers that embed a TEDS.

DALI devices are very simple in nature and don't support the ability to embed a TEDS in the devices. Nevertheless a TEDS for the DALI devices can still be used, but in this case it would be embedded in a DALI gateway as shown in Figure 5. Note that in this scenario, the STIM (Smart Transducer Interface Module) that would normally reside in the device resides in the gateway. STIMs that are implemented in this way are referred to as "Soft STIMs". 
Note that devices with identical functionality on the DALI and IBECS 1-Wire, PLC, and RF networks can be represented in a common fashion to any applications that need to interface to both. The TEDS that exist in the DALI gateway can be created dynamically as devices on the DALI network are discovered. It is worth noting that the many attributes of the TEDS shown above would be the same whether they resided on a DALI gateway or are embedded in the devices.

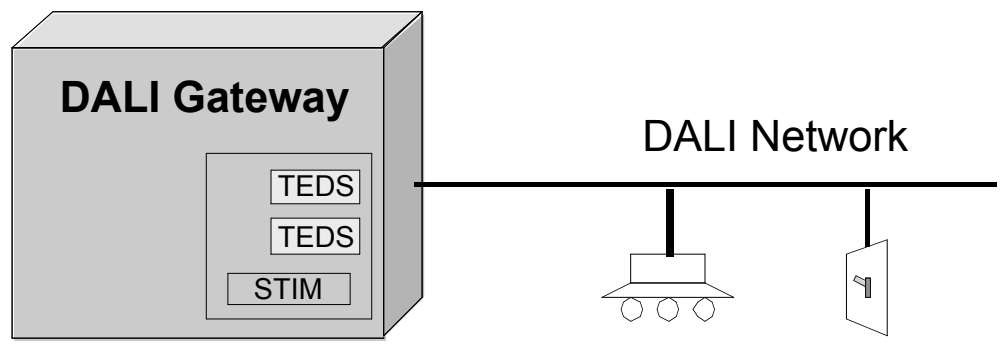

Figure 5. A simple DALI gateway, with virtual TEDS and STIMs residing in the gateway rather than in the DALI device itself.

In addition to using the TEDS as described above, it is possible to use the specifications from IEEE 1451.1 to expose an object oriented interface to the devices of the IBECS 1Wire, DALI, RF, and PLC networks. IEEE 1451.1 defines the concept of a Network Capable Applications Processor (NCAP). An NCAP could be implemented as part of a gateway that provides interfaces to all of the relevant networks as shown in Figure 6.

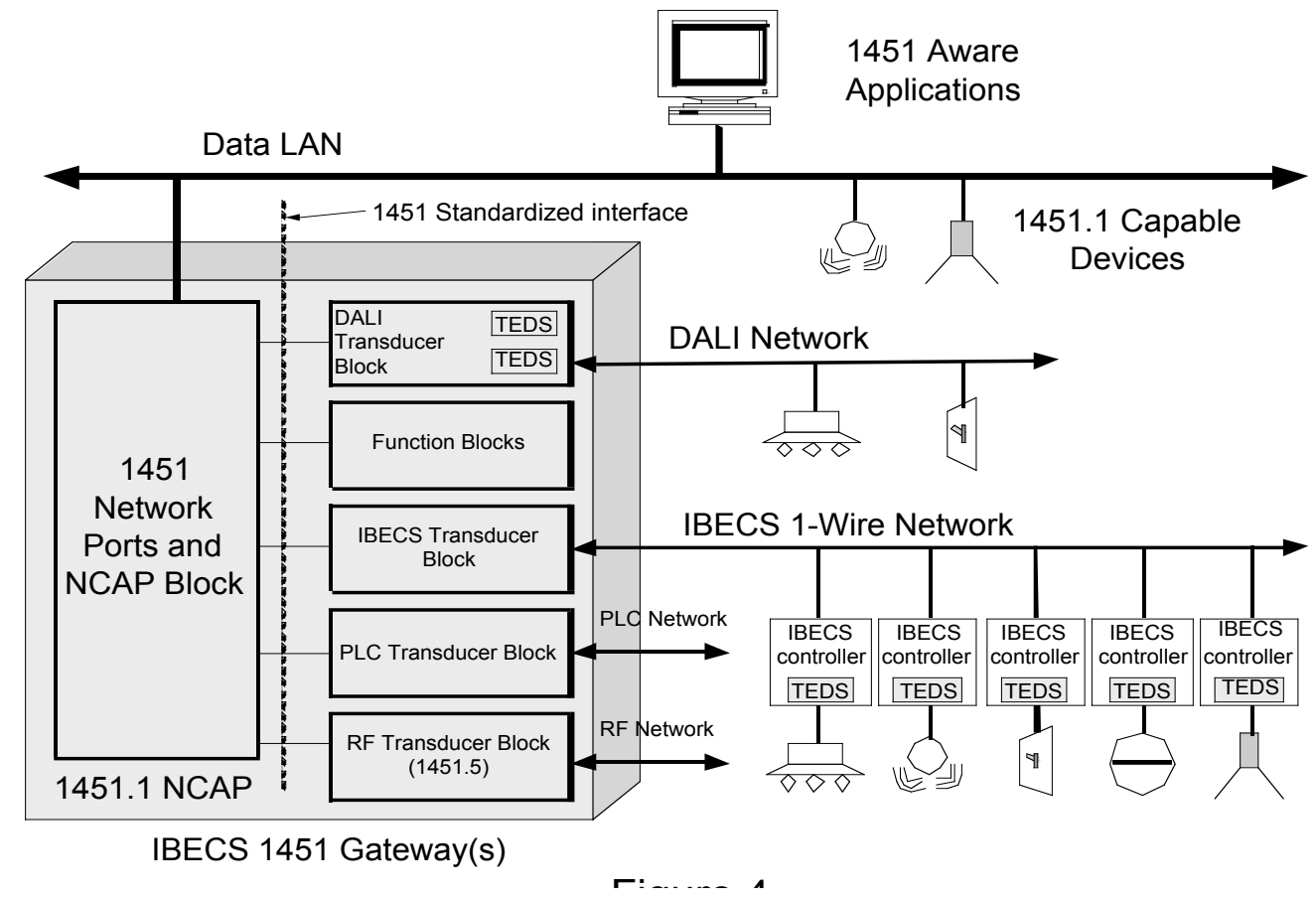

Figure 6. Generalized system diagram showing the NCAP (Network Capable Applications Processor) on the IBECS 1451 gateway and how communications between the NCAP and the different network blocks is mediated through a standardized 1451 interface. 
Although the gateway in Figure 6 is depicted as a single entity, it should be understood that there may actually be separate gateways for each type of DAN network. There is no requirement that all the DAN interfaces exist within a single box.

Within the NCAP are defined a number of software components including:

- A standardized 1451 interface that provides a common API for all applications that need to communicate with the DAN devices.

- NCAP Block used to provide the 1451.1 "plumbing". This block facilitates communications between all the other blocks in the system. These are implemented with a Block type class.

- Transducer Block used to communicate to the various transducer communications channels in the system. In the scenario described, there would be a transducer block for DALI and a transducer block for IBECS. The TEDS for the devices on a DALI network would exist within the DALI transducer block. These are implemented with a Block type class.

- Function Block for implementing local application level logic. These are implemented with a Block type class.

- Network accessible variables and parameters. These provide a level of programmability by applications. These are implemented with a Component type class.

- Network communications port. These provide access to applications on the LAN that want to communicate with the NCAP. These are implemented with a Service type class.

The transducer blocks provide means to communicate with the devices on the DALI and IBECS networks and the TEDS publishes the capabilities of the devices on those networks. The function blocks within the NCAP provide local logic and implement the object interface classes that allow applications on the LAN to access the functionality of the devices and the NCAP. The applications on the LAN can communicate with the NCAP using either a client/server (tightly coupled) model or by using a publish/subscribe (loosely coupled) model. Furthermore, 1451.1 provides a mechanism for the applications on the LAN to discover NCAPs and their capabilities. Examples of these applications include facility monitoring or HVAC control programs.

Also, note that the 1451.1 interface allows the integration of other 1451.1 capable devices on the LAN network. Examples of such devices are made by companies such as Esensors Inc. (http://www.eesensors.com/).

One example is a dimmable ballast on a DALI network and an occupancy sensor on the IBECS 1-Wire network where both networks are attached to an NCAP gateway as shown in figure 6. TEDS and transducer blocks exist as shown. Furthermore, the following function blocks are assumed within the NCAP:

- Function block for controlling dimmable lights that defines a class with the following methods for setting and getting the state of a light.

○ SetLightLevel(light_ID, light_level)

○ GetLightLevel(light_ID) 
- Function block for getting the state of an occupancy sensor that defines a class with the following methods.

- GetState(occupancy_ID) - this method

○ PublishState(occupancy_ID)

- Function block for implementing local logic that defines a class with the following methods.

○ SetTriggerEvent(trigger)

$\circ \operatorname{Set} A c t i o n($ action)

In this example, there are general classes that access lights and sensors by specifying an ID. Another way to implement this would be to instantiate a separate object instance for each instance of a light or sensor. From an API point of view, both of these approaches are equivalent since in both cases it is necessary for the application to refer to specific lights or sensors. Also, the state of the occupancy sensor can be obtained in two ways. The first is by simply requesting the state. The second is to configure the sensor to publish its information using the publish/subscribe mechanism supported by 1451.1. The publish subscribe mechanism provides a means for the occupancy sensor's state to be broadcast whenever it changes and allows for asynchronous events to drive logic in other parts of the system.

The local logic function block might be implemented in a number of different ways including using some sort of interpretive language (Java, Tcl, Perl, Python) and downloading code or parameterizing simple events and coupling them with simple actions. The amount of flexibility required typically reflects the range of functionality provided by the devices and the range of functionality needed by the NCAP. For simplicity, the later is depicted where one can specify a simple event such as:

Occupancy_sensor_2 = TRUE

And a simple action such as:

Turn light_3 to level 255

Using these APIs, it would be possible for applications to get and set the state of the light, and query the state of the occupancy sensor. If so configured, the occupancy sensor could also publish its state whenever it changes, and if any applications have subscribed to receive it, they would also get the state of the occupancy sensor. Furthermore, it is possible to configure the NCAP with local logic such that a light may be turned on if an occupancy sensor is triggered AND the state of the occupancy sensor can also be published to other applications as described above. This allows for a great deal of flexibility as to where the system level logic is implemented.

\section{Role of BACnet}

IEEE 1451.1 provides a standard framework and mechanism for implementing objectoriented APIs, but what it lacks is a standard set of industry specific objects that would go even further in facilitating interoperability and application development. BACnet provides a simple set of pre-defined objects and is more prevalent in building automation 
than is 1451. There are an increasing number of applications and tools that utilize BACnet. In addition, Annex J of the BACnet protocol allows for using BACnet over IP networks, also referred to as BACnet/IP. Therefore it may be desirable to combine elements of BACnet and 1451 in a Unified Framework Gateway as shown in Figure 7. In the architecture shown in Figure 7, the application object interface in the gateway is compliant with BACnet so that applications that speak BACnet can interface and use the functionality of the devices. Like 1451, BACnet supports the notion of both a tightly and loosely coupled interface. Also, the same 1451 transducer blocks and API that was shown in figure 6 can be re-used to provide a method for interfacing to the various DAN networks. As before, the TEDS provide for a way to discover devices and instantiate BACnet objects that can be accessed by the BACnet applications.

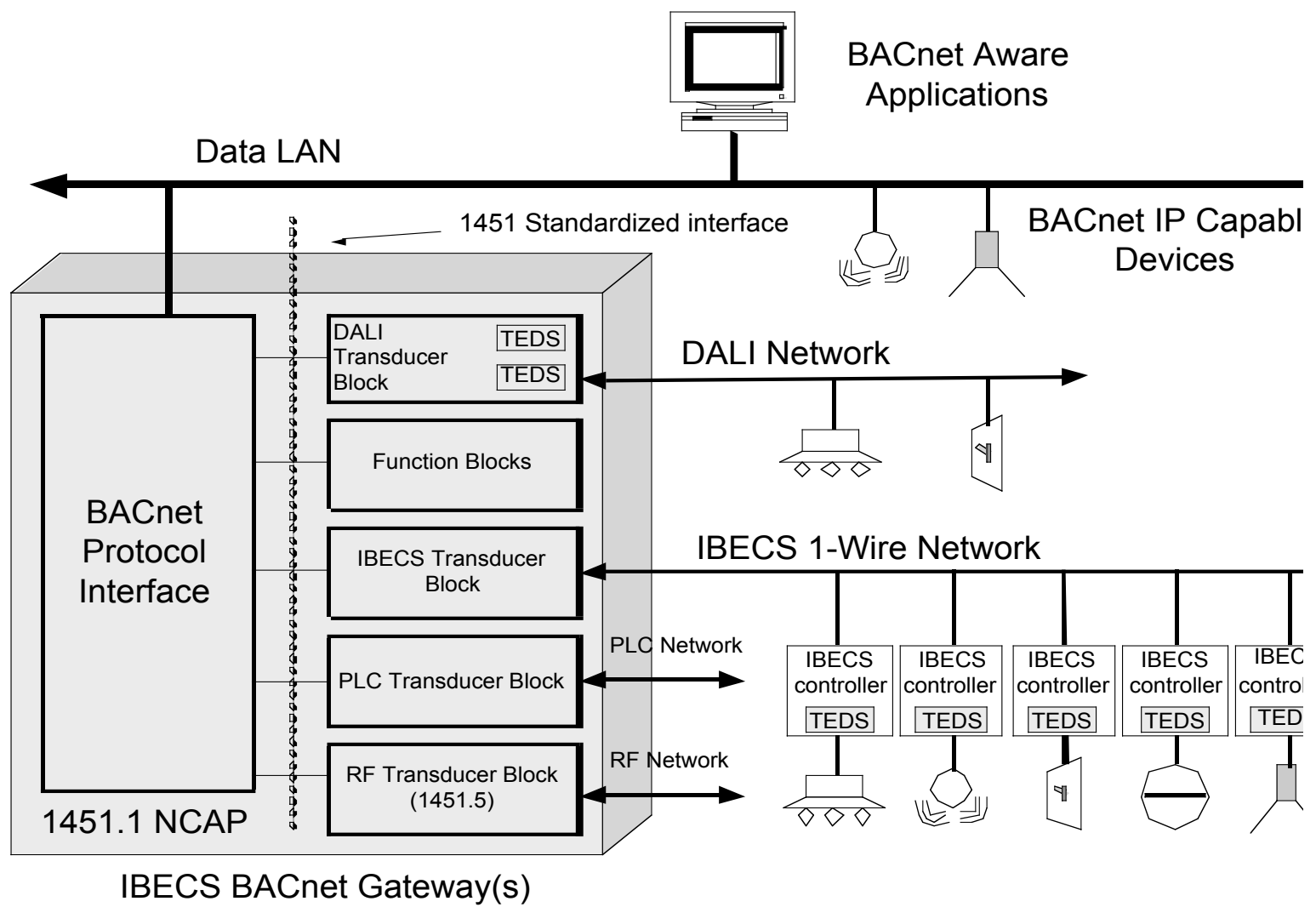

Figure 7. Generalized system diagram showing how a BACnet aware application can control both BACnet IP capable devices directly on the data LAN as well as networked devices on DALI, IBECS, PLC, or RF networks via the IBECS BACnet gateway and the 1451 standardized interface.

As a final scenario, it is possible to incorporate 1451.1 and BACnet into a common gateway as shown in figure 8 . This would provide the maximum in flexibility and allow the integration of both BACnet and 1451.1 compliant devices over the IP network. 


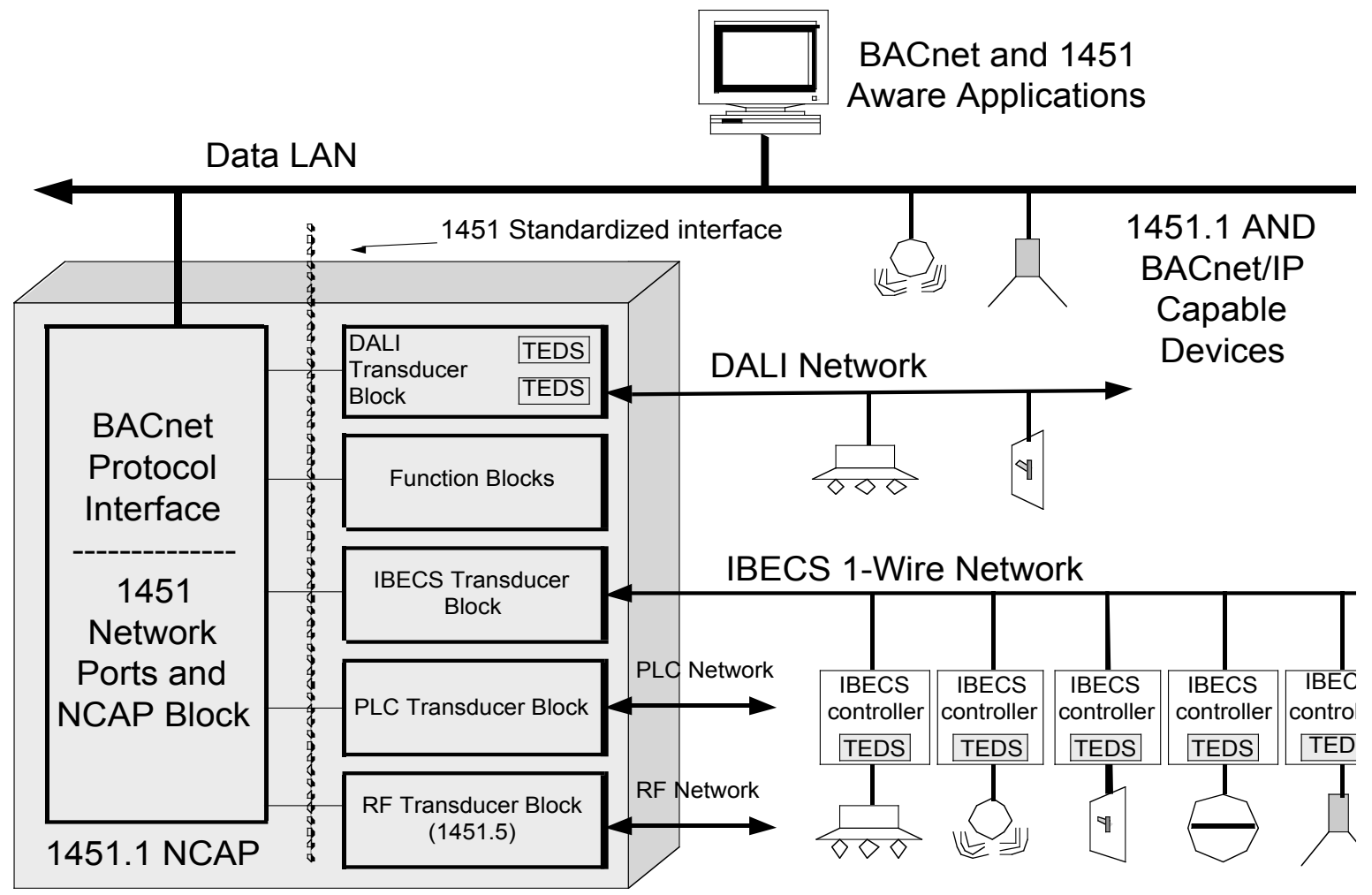

IBECS Unified Framework Gateway(s)

Figure 8. Generalized system diagram showing how BACnet and 1451-aware application can control both BACnet IP and 1451.1 devices directly on the data LAN as well as networked devices on DALI, IBECS, PLC, or RF networks via the IBECS BACnet gateway and the 1451 standardized interface.

\section{Conclusion}

Many lighting control companies have robust local lighting control systems with functional strategies for the office level controls. What the industry lacks is the efficient integration of local controls with building controls and energy management systems in order to utilize sensory data. LBNL proposes a framework for a gateway with a level of embedded intelligence, linking various device area networks (DANs) to building control systems. The proposed gateway acts as a translator for DANs enabling them to talk to each other and with a building control system. Just like a PC recognizing a mouse as soon as it is plugged in, the gateway will recognize devices with embedded or virtual TEDS. This presents a truly "plug and play" capability for the building control systems. As a result, sensory data can be automatically calibrated, collected and utilized with minimal labor for effective and efficient building controls.

The mature market cost of the proposed gateway is not yet known. But current product suggest that $\$ 1000 /$ bridge is achievable today with off-the-shelf components and wireless routers that are common today for computer applications do not have significantly lower capabilities than the $\$ 1000$ product and are now available at $\$ 100-\$ 200$. If it is configured so that it caters to commissioning, maintenance and energy monitoring, its 
benefit from a mere energy saving equipment can be extended to reduction in installation and maintenance costs.

\section{Future Directions}

One of the main features of note in the gateway scenarios discussed above is that they all leverage 1451 to interface to the DAN networks in order to provide a consolidated API that can be used by other function blocks and LAN application interfaces in the gateway. Therefore, further work should concentrate on developing an appropriate version of this API for lighting control applications. Initial work should focus on the DALI and IBECS 1-Wire networks with RF and PLC to follow. The LAN application of choice should be based upon BACnet to provide consistency and continuity with previous work in building automation protocols. The following are a brief survey of the tasks involved in future work.

- Increase involvement with the 1451 standards effort. More specifically, support the newly established JDDAC development effort, which combines 1451 with Java for data acquisition and control applications. Involvement in this effort will allow LBNL to leverage their work to implement a gateway.

- Choose a platform for developing and prototyping a gateway targeted for building control. Alternatives for this effort include the TINI bridge from Maxim Dallas Semiconductor.

- Design appropriate TEDS, function blocks, and APIs for the gateway.

- Choose a prototypical off the shelf BACnet application for testing.

- Develop a prototype hardware interface for the DALI and 1-Wire networks.

- Implement a prototype of the device STIMs and function blocks to provide the standardized API.

- Develop a BACnet interface block for the gateway.

- Demonstrate DALI and IBECS 1-Wire working with a BACnet application.

- Publish API specifications and reference implementation to encourage adoption.

\section{References}

[1] F. M. Rubinstein, S. Treado, and P. Pettler, "Standardizing Communication Between Lighting Control Devices: a Role for IEEE P1451," presented at IEEE-IAS Annual Conference, Salt Lake City, Utah, 2003.

[2] F. M. Rubinstein and P. Pettler, "Final Report on Internet Addressable Light Switch," LBNL-49974, August, 20012001 